\title{
Motor learning of a dynamic balance task: Influence of lower limb power and prior balance practice
}

\author{
Louis-Solal Giboin*, Markus Gruber, Andreas Kramer \\ Sensorimotor Performance Lab, University of Konstanz, Germany
}

Keywords:

Balance training

Specificity

Leg strength

Fall prevention

Learning to learn

\begin{abstract}
A B S T R A C T
Objectives: We wanted to verify if the "learning to learn" effect observed in the learning of visuomotor tasks is also present when learning a balance task, i.e., whether the learning rate of a balance task is improved by prior practice of similar balance tasks.

Design: Single centre, parallel group, controlled training study.

Methods: 32 young healthy participants were divided into a control and a training group. The training group's practice consisted of 90 trials of three balance tasks. Forty-eight hours after the training, we recorded performance during the acquisition (90 trials) of a novel balance task in both groups, and $24 \mathrm{~h}$ thereafter we measured its retention (10 trials).

Results: Mixed models statistical analysis showed that the learning rate of both the acquisition and the retention phase was not influenced by the 90 prior practice trials performed by the training group. However, participants with high lower limb power had a higher balance performance than participants with low power, which can be partly explained by the higher learning rate observed during the acquisition phase for participants with high power.

Conclusions: Contrary to visuomotor or perceptual tasks, we did not find a "learning to learn" effect for balance tasks. The correlation between learning rate and lower limb power suggests that motor learning of dynamic balance tasks may depend on the physical capability to execute the correct movement. Thus, a prior strength and conditioning program with emphasis on lower limb power should be considered when designing a balance training, especially in fall prevention.
\end{abstract}

\section{Introduction}

Short-term balance training induces task-specific performance improvement, ${ }^{1-3}$ and consequently balance should be seen more as a sum of task-specific skills than a general ability. ${ }^{2}$ Therefore, in regard to fall prevention, which is a major health and resource issue, ${ }^{4,5}$ patients should learn as many balance tasks as possible, and as close as possible to real-life fall contexts. ${ }^{2}$ The implementation of this recommendation requires optimization of balance task learning, especially in the setting of clinical rehabilitation where there is limited time to practice. The learning rate of a novel motor task can be improved with previous learning experience of relatively similar tasks. ${ }^{6}$ This phenomenon has been termed "learning to learn". ${ }^{7}$ One of the several possible mechanisms driving this

Abbreviations: $\mathrm{P}_{\max }$ rel, maximal power relative to bodyweight measured during a counter movement jump.

* Corresponding author.

E-mail address: louis-solal.giboin@uni-konstanz.de (L.-S. Giboin). effect is called structural learning, where invariants of several tasks sharing the same structure are extracted and facilitate the learning of a new task also sharing the same structure. ${ }^{8}$ The "learning to learn" effect has been demonstrated for cognitive ${ }^{9-11}$ and visuomotor tasks, 6,12 including a locomotion task relying on distorted visual feedback. ${ }^{13}$ To our knowledge, this "learning to learn" effect has never been specifically tested for complex full-body tasks such as balance tasks. This learning facilitation would be of interest to reduce the time to learn many different balance tasks. We hypothesized that prior practice of similar balance tasks improves the learning rate of a novel balance task.

However, contrary to visuomotor or perceptual tasks, performance during balance tasks might not only depend on the optimization of the motor command or strategy, but also on the physical capacity to adequately perform the movement. In particular, some studies have reported an influence of lower limb strength and power on balance performance, although this finding is challenged by the results of other studies reporting no significant correlation. ${ }^{14,15}$ To shed light on this issue, we also measured lower limb power and assessed its influence on the learning rate. 


\section{Methods}

32 young healthy adults ( 15 women, 17 men, age $=24 \pm 3$ years; height $=172 \pm 9 \mathrm{~cm}$; weight $=68 \pm 14 \mathrm{~kg}$ ) participated in the study after giving written informed consent. The experiment was in accordance with the regulations of the ethics committee of the University of Konstanz as well as the Declaration of Helsinki. Subjects were moderately active students that did not regularly engage in balance training or related activities, were naive to the tested and trained balance tasks, and free of lower limb injuries or impairments related to balance. Moreover, they were asked to perform absolutely no balance training during the whole study duration and no heavy leg training $48 \mathrm{~h}$ prior to any experimental sessions.

Subjects participated in 3 experimental sessions, the first one consisting of pre tests and prior practice for the training group, the second consisting of the acquisition phase, and the third one of the retention test. In the first experimental session, subjects performed 3 maximal countermovement jumps with 1 min of rest in between jumps, hands on the hips, on a force plate (Leonardo Mechanograph). Then, we measured their pre-training performance on the tilt-board task (3 trials). There was no warm-up or familiarization with the device prior to the measurements. We matched subjects in a control ( $\mathrm{N}=16,9$ women) and a training group $(\mathrm{N}=16,6$ women) according to their performance in the first tiltboard task trial and their maximal power relative to body weight ( $P_{\max }$ rel) exerted during the counter movement jumps (first trial: control $=0.42 \pm 0.7 \mathrm{~s}$, training $=0.43 \pm 0.4 \mathrm{~s}$, p-value $=0.97 ; \mathrm{P}_{\max }$ rel: control $=45 \pm 9 \mathrm{~W} / \mathrm{kg}$, training $=45 \pm 8 \mathrm{~W} / \mathrm{kg}$, p-value $=0.83$, which corresponded to a jump height of $38 \pm 11 \mathrm{~cm}$ for control and $40 \pm 8 \mathrm{~cm}$ for training, $\mathrm{p}=0.61$ ). To be more precise, the firsts 10 participants were randomly allocated to one of the groups, and the following participants were assigned with the intention to match group performance with respect to tilt-board performance and $P_{\max }$ rel. Ten minutes after this pre-test, subjects from the training group practiced 3 different balance tasks with 30 trials each. Subjects assigned to the control group did not practice. Forty-eight hours later, subjects from both groups came back to the laboratory to perform the novel tilt-board task (90 trials, acquisition phase). Twenty-four hours later, we measured again the performance of subjects from both groups on the tilt-board task (10 trials, retention phase).

For all balance tasks, an acoustic signal was given to indicate when to get ready for the trial ( $3 \mathrm{~s}$ before), when to start and when to finish the trial. Every trial lasted $10 \mathrm{~s}$, and there was a break of 1 min every 10 trials. Before starting any of the four balance tasks, the subject was instructed where to put the foot on the device. During the practice, but also during the pre-training, acquisition and retention phases, we gave the subject an estimated time in equilibrium for every trial (estimation done with a stop watch).

The tilt-board task consisted of a one-leg stance (with the preferred leg) on a tilt-board with a medio-lateral axis of perturbation, hands on the hips ( $\mathrm{see}^{2}$ for details). The instruction was as follows: (i) start with the preferred foot on the board, the other foot, i.e. the foot of the free leg, on the floor, with the tilt-board side opposite to the free leg on the floor in a stable position. (ii) Lift the free foot from the floor and then bring the board into a horizontal position for as long as possible. (iii) If you lose equilibrium or touch the floor with the free leg while the trial is not over yet, restart from the initial position. We fixed a motion capture marker on the big toe's nail of the free leg to be able to exclude phases with foot support during the offline analyses.

The 3 practice tasks were all one-leg stance tasks done with the same leg and the same procedure as the tilt-board task. The 3 tasks consisted of balancing on a slack line $(5 \mathrm{~m}$ long, $3 \mathrm{~cm}$ wide, Slackline Tools, medio-lateral axis of perturbation), a BOSU ball (BOSU balance trainer, perturbation in all directions) and a differ- ent tilt-board (Sensoboard, Sensosports $\mathrm{GmbH}$; perturbations in all directions; the longer side of the board in the antero-posterior axis of the subject).

The prior practice was done serially on the slack line, BOSU ball and Sensoboard (i.e. one trial on the slack line, then one trial on the BOSU ball, on trial on the Sensoboard, and so on). The order of the tasks was counter-balanced between subjects. The practice consisted of a total of 90 trials ( 30 trials for each task).

$P_{\max }$ rel was defined as the highest value among the three countermovement jumps performed. Power was calculated as the product of force and velocity (derived from changes in force) with the Leonardo GRFP 4.3 software.

We measured the tilt-board task performance with motioncapture (Vicon Nexus, 12 T40s cameras, $200 \mathrm{~Hz}$ ). In brief, performance corresponded to the time (in s) where the platform of the tilt-board was in equilibrium, i.e. positioned parallel to the floor (with a margin of $\pm 5^{\circ}$ ). Phases where the free foot touched the ground were subtracted from the time at equilibrium. In addition, any time at equilibrium achieved by getting into equilibrium via the free foot on the ground was neglected.

Statistics were done with R (R 3.4.2, the R foundation for statistical computing) and JASP (JASP 0.8.6, University of Amsterdam). We used linear mixed effects models (lme4 R package ${ }^{16}$ ) and the Satterthwaite's method to approximate degrees of freedom and obtain p-values (lmerTest R package ${ }^{17}$ ). To test the effect of a group factor (e.g. training vs. control groups) on the learning rate of the balance task, we needed to test the effect of each group on the slope of performance across trials. Therefore, in a model with random intercepts for subjects, we have tested the interaction between groups and number of trials. A t-test was used to compare height, weight and power between men and women. We performed Pearson correlations between $P_{\max }$ rel and the first trial of the pre and retention phases, height and weight. Additionally, we tested whether there was a difference in the first trial of each phase between groups (low $\mathrm{P}_{\max }$ Rel and high $\mathrm{P}_{\max }$ Rel) with a repeated measures ANOVA. When sphericity was violated, we used a Greenhouse-Geisser correction. We performed Bonferroni corrected t-tests between groups at each phase level as post-hoc tests.

\section{Results}

Mixed models analyses revealed no significant differences between the training and control groups' estimates of the mean performance and the slope of performance progress across trials (Table 1). This indicates that there was no difference between groups in the overall balance performance and learning rate (see Fig. 1A).

Lower limb power $\left(\mathrm{P}_{\max } \mathrm{rel}\right)$ correlated with the performance of the first trial in the retention test $(r=0.60, p<0.001)$ and the average of performance in the retention test $(0.651, \mathrm{p}<0.001)$ but not with the performance of the first trial of the pre phase $(r=0.186$, $\mathrm{p}=0.308$ ), indicating a possible effect of power on the learning rate. To look into this correlation in more detail, we divided the subjects into two groups according to their $\mathrm{P}_{\max }$ rel, one group with $P_{\max }$ rel higher than the median (High $P_{\max }$ rel) and the other group lower (Low $P_{\max }$ rel) than the median (median $=44.8 \mathrm{~W} / \mathrm{kg}$, see Fig. 1B; for individual examples of the two groups, see Fig. 1C and $\mathrm{D}$, respectively). Mixed model analysis revealed that averaged balance performance in the pre trials was not significantly different between high and low power groups. In the acquisition and retention phase, the estimate of the mean of balance performance was twice as high in the high power group than in the low power group (Table 1). This higher mean performance might be related to a higher balance performance in the first trial of each phase. An ANOVA performed on the first trial of each phase showed a 
Table 1

Mixed effects models results for training groups and power groups. Estimates of the slopes of balance performance across trials (in s) and estimates of the balance performance mean (in s) of each group in the 3 phases of the study (pre, acquisition and retention). SE corresponds to standard error. High P and low P represent groups with high and low $\mathrm{P}_{\max }$ rel respectively. Control group, training group, high $\mathrm{P}$ and low $\mathrm{P}$ estimates represent the mean performance per group. The interaction estimates represent the slope of performance across trials. Control group: trial, control group, high P: trial, and high P estimates are tested against zero. Training group: trial, training group, low P: trial, and low P estimates and are tested against the estimate of the other group. *, **, and ${ }^{* * *}$ indicate a p-Value inferior to $0.05,0.01$ and 0.001 respectively.

\begin{tabular}{|c|c|c|c|c|c|}
\hline DV & Phase & Fixed effect & Estimate (SE) & t-Value (df) & p-Value \\
\hline \multirow[t]{24}{*}{ Performance } & \multirow[t]{4}{*}{ Pre } & Control group: trial & $0.16(0.08)$ & $2.06(62)$ & $0.044^{*}$ \\
\hline & & Training group: trial & $0.15(0.11)$ & $-0.05(62)$ & 0.96 \\
\hline & & Control group & $0.31(0.21)$ & $1.48(84.3)$ & 0.142 \\
\hline & & Training group & $0.29(0.3)$ & $-0.08(84.3)$ & 0.93 \\
\hline & \multirow[t]{4}{*}{ Acquisition } & Control group: trial & $0.018(0.0013)$ & $13.5(2846)$ & $2 \mathrm{e}-16^{* * *}$ \\
\hline & & Training group: trial & $0.017(0.0018)$ & $-0.24(2846)$ & 0.59 \\
\hline & & Control group & $1.57(0.27)$ & $5.7(33)$ & $2.3 e-06^{* * *}$ \\
\hline & & Training group & $1.36(0.39)$ & $-0.53(33)$ & 0.59 \\
\hline & \multirow[t]{4}{*}{ Retention } & Control group: trial & $0.14(0.04)$ & $3.5(286)$ & $5 e-03^{* * *}$ \\
\hline & & Training group: trial & $0.13(0.06)$ & $0.17(286)$ & 0.87 \\
\hline & & Control group & $2.39(0.41)$ & $5.8(47)$ & $5.37 \mathrm{e}-07^{* * *}$ \\
\hline & & Training group & $2.15(0.58)$ & $-0.42(47)$ & 0.67 \\
\hline & \multirow[t]{4}{*}{ Pre } & High P: trial & $0.15(0.07)$ & $1.95(62)$ & 0.054 \\
\hline & & Low P: trial & $0.16(0.1)$ & $0.08(62)$ & 0.93 \\
\hline & & High P & $0.55(0.2)$ & $2.72(88.6)$ & $0.007^{* *}$ \\
\hline & & Low P & $0.05(0.28)$ & $-1.74(88.6)$ & 0.08 \\
\hline & \multirow[t]{4}{*}{ Acquisition } & High P: trial & $0.022(0.001)$ & $16.5(2846)$ & $2 \mathrm{e}-16^{* * *}$ \\
\hline & & Low P: trial & $0.014(0.002)$ & $-4.39(2846)$ & $1.16 \mathrm{e}-05^{* * *}$ \\
\hline & & High P & $1.94(0.21)$ & $8.8(34.8)$ & $1.9 \mathrm{e}-10^{* * *}$ \\
\hline & & Low P & $1(0.31)$ & $-3(34.8)$ & $0.004^{* *}$ \\
\hline & \multirow[t]{4}{*}{ Retention } & High P: trial & $0.17(0.04)$ & $4.24(286)$ & $2.9 \mathrm{e}-05^{* * *}$ \\
\hline & & Low P: trial & $0.12(0.06)$ & $-0.88(286)$ & 0.37 \\
\hline & & High P & $3.02(0.34)$ & $8.73(58.2)$ & $3.5 \mathrm{e}-12^{* * *}$ \\
\hline & & Low P & $1.51(0.48)$ & $-3.1(58.2)$ & $0.003^{* *}$ \\
\hline
\end{tabular}

phase effect $(\mathrm{F}(2,60)=20.6, \mathrm{p}=0.002)$, a group effect $(\mathrm{F}(1,30)=17.4$, $\mathrm{p}<0.001)$, and a group $\times$ phase interaction $(\mathrm{F}(2,60)=4.9, \mathrm{p}=0.016)$. Post-hoc corrected t-tests indicated that performance was indeed higher for the group with high $\mathrm{P}_{\max }$ rel in the retention phase (High $P_{\max }$ rel $=2.23 \pm 1.2 \mathrm{~s}$, Low $P_{\max }$ rel $=0.84 \pm 0.8 \mathrm{~s}, \mathrm{t}(30)=3.8$, $\mathrm{p}<0.001$ ) but not in the pre phase (High $\mathrm{P}_{\max }$ rel $=0.62 \pm 0.68 \mathrm{~s}$, Low $\left.\mathrm{P}_{\max } \mathrm{rel}=0.23 \pm 0.28 \mathrm{~s}, \mathrm{t}(30)=2.09, \mathrm{p}=0.13\right)$ or acquisition phase (High $P_{\max }$ rel $=0.89 \pm 0.48 \mathrm{~s}$, Low $\mathrm{P}_{\max }$ rel $=0.47 \pm 0.83 \mathrm{~s}, \mathrm{t}(30)=1.7$, $\mathrm{p}=0.27$ ). The higher mean performance seen with the mixed model analysis is also explained by the two times faster learning rate observed during the acquisition phase for the group with high power (Table 1).

\section{Discussion}

The results of the present study show that one practice session consisting of several balance tasks had no influence on the performance and learning rate of a novel balance task. However, lower limb power was a predictor of learning rate and balance performance during the acquisition phase and of the balance performance measured during the retention of the novel balance task.

One could argue that the fact that we found no evidence of the learning to learn effect was simply due to methodological issues. However, we took great care to design our experiment with the aim to facilitate the "learning to learn" effect. For this purpose, we had a similar experimental structure as previously used, ${ }^{6}$ the prior practice was done with various tasks as it should facilitate "learning to learn" effect, ${ }^{12}$ and we used practice and testing tasks sharing the same structure (very similar body position, shared axis of perturbation, same movement sequence order for all tasks) as it should facilitate structural learning., $, 9,12$ Despite these experimental precautions, we could not detect any facilitation effect of the previous balance practice session on the learning rate of the tested task. It could be argued that the practice volume, although similar to the one used in a previous study, ${ }^{6}$ was not large enough to facilitate following learning. However, we have seen in the present study that 90 specific trials increased the learning rate tenfold on the following day (see Table 1, acquisition vs. retention). Thus, if 90 non-specific trials are not enough to induce a noticeable effect on the learning rate, the "learning to learn" effect for dynamic balance tasks is, if existent, quite weak and probably not relevant in a clinical context. This lends further support to the task specificity principle of balance training that has been observed in recent studies. ${ }^{2,3,18}$ It is still possible that a very large prior training volume could induce a learning to learn effect, as documented for example by the influence of expertise level of video game players on the learning rate of new video games. ${ }^{10}$ Thus, a "learning to learn" effect in expert balance tasks practitioners (e.g. professional slack liners) could possibly exist and be important for athletic performance in new situations. We speculate that the diverging results concerning the "learning to learn" effect for arm movements traditionally used in motor learning studies and our tested balance task stems at least partly from the different movement complexity and the different neural substrates controlling both types of movement.

We observed that lower limb power was a good predictor of the performance during the retention test. The higher performance can be explained by a slightly higher performance at the beginning of the acquisition phase, and by the much faster learning rate associated with higher lower limb power. If this is truly a causative relation, it is possible that the higher power allows more successful attempts, a movement execution closer to the one planned, and more accurate online corrections, which probably positively affect the learning rate by small but cumulative increments throughout the learning trials.

It is known that muscle function, i.e. strength and power production capacity, is a determinant factor of postural control and balance performance especially in populations with reduced muscle function. ${ }^{19,20}$ However, in healthy populations, this relation is less clear. For example, in healthy adults of all ages no relationship could be found between balance performance and muscle function, ${ }^{21-23}$ and strength training seems to have no effect on postural control. ${ }^{24}$ On the other hand, in other studies, a power training intervention increased balance performance in healthy old adults and in adolescents. ${ }^{25,26}$ It has been proposed that once a certain strength or power threshold is reached, the additional strength or power is not beneficial to the balance performance anymore. ${ }^{15}$ Our 

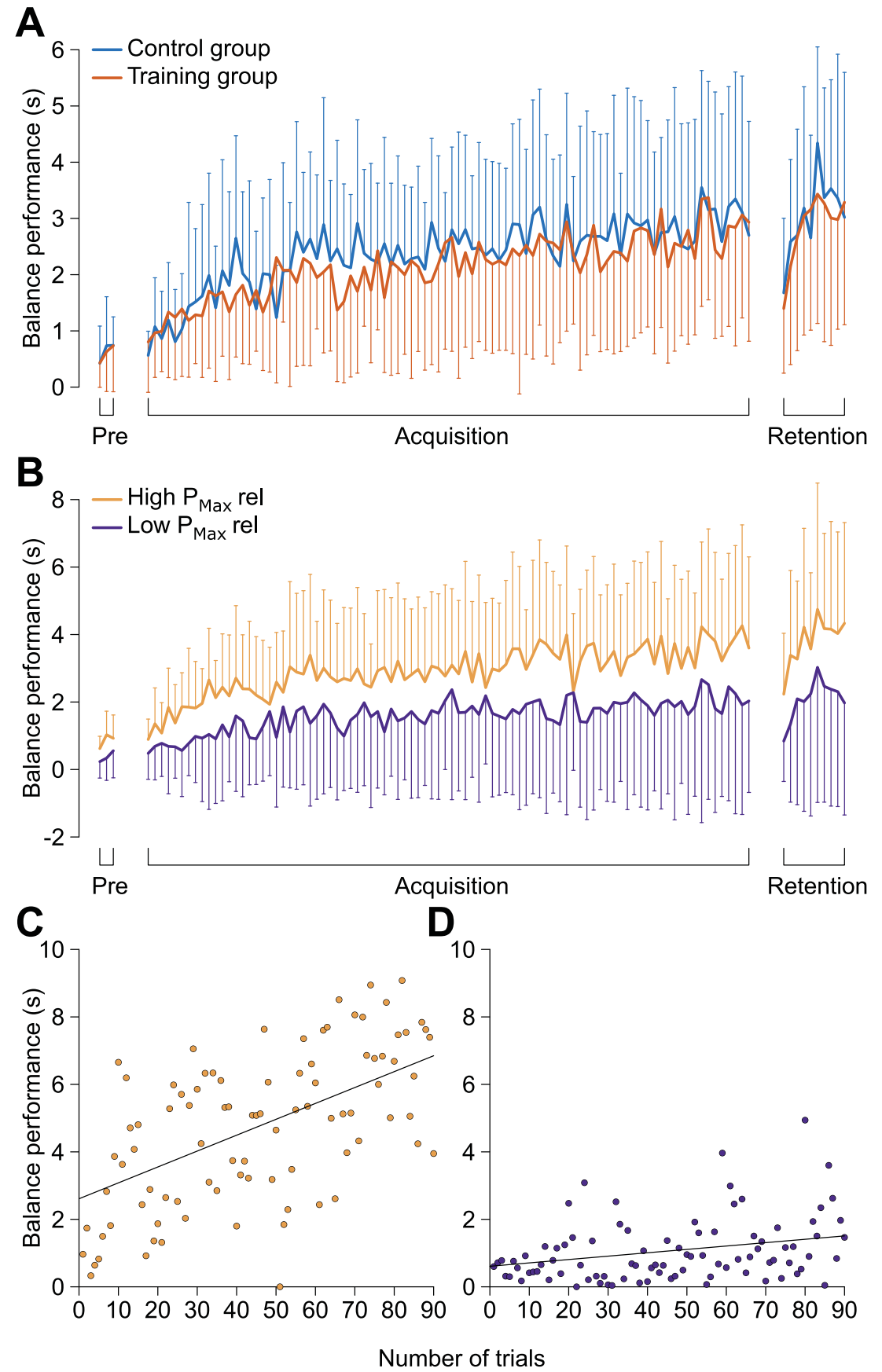

Fig. 1. Learning rate of the tilt-board task. A and B. Averaged performance (in s) of the tilt-board task during the pre-training (Pre, 3 trials), acquisition (90 trials) and retention (10 trials) phases. (A) Performance of the training (red) and control (blue) group. (B) Performance of the group with higher $P_{\max }$ rel (orange, High $P_{\max }$ rel) than the median $\mathrm{P}_{\max }$ rel and of the group with a lower $\mathrm{P}_{\max }$ rel (purple, Low $\mathrm{P}_{\max }$ rel) than the median $\mathrm{P}_{\max }$ rel. Error bars correspond to standard deviation. C and D. Performance (in $\mathrm{s}$ ) during each trial for a representative individual from the high (C) a low (D) $P_{\max }$ rel groups. (For interpretation of the references to color in this figure legend, the reader is referred to the web version of this article.)

results suggest that this proposition describes only partially the relationship between power and balance performance. Our subjects were most probably above the power threshold suggested by Paillard. ${ }^{15}$ In line with Paillard's threshold proposition, we have seen no correlation between power and the performance during the first trial, but performance difference could be seen when grouping participants in a high and low power group. We can conclude that when power is above a certain threshold, it may have a limited effect on the balance performance of the first, or first few, trials of a new balance task. However, contrary to Paillard's proposition, power was an important predictor of the balance task's learning rate during the acquisition and correlated well with the retention. The moderate effect of power on the first trial and the strong influence of power on the learning rate may explain the literature's discrepancies. Indeed, depending on the number of trials realized during the tests of balance performance, power may or may not influence the average of performance among trials. This effect most probably interacts more or less depending on how close the tested population muscle function is to the impairment threshold and how much the tested balance task relies on strength or power. ${ }^{15}$ Similarly, the strong effect of power on the learning rate could confound a transfer or a "learning to learn" effect that can be seen after bal- 
ance training, especially in population with low pre-training power capacity. Therefore, as suggested by Kattner et al. for perceptual tasks, ${ }^{9}$ the effect of a balance training should not be interpreted solely with the average of few trials performance. A transfer effect should be interpreted only with the first or first few trials comparison, and the effect of an intervention on balance performance should be assessed by taking the learning curve into account. Moreover, in any balance training studies, lower limb power assessments should be included, as its possible increase by the training could explain the increase in balance performance as much as the balance training itself.

It should be noted though that the relation between the performance and the lower limb power is not necessarily a causative one. A training study observing the effects of an increase in lower limb power on the learning rate is necessary to answer this question. It is possible that the relationship we observed between lower limb power and learning rate is spurious and that the real predictor of the learning rate is not $\mathrm{P}_{\max }$ rel but for instance the rate of force development of the lower limb muscles, or other biomechanical or anthropometric parameters. For example, it has been shown that rate of force development can be used to distinguish elderly fallers and non-fallers, ${ }^{27}$ and balance training has been shown to increase rate of force development. ${ }^{28}$ Nevertheless, it seems reasonable to state that the physical capability to perform the correct movement - whether this is best characterized by lower limb power or other correlated variables - plays an important role in the acquisition and retention of a dynamic balance task. Whether this effect is as pronounced in more static balance tasks requiring less power than dynamic balance tasks is an interesting question that warrants further investigation.

\section{Conclusion}

We observed no "learning to learn" effect for balance task learning. Thus, prior short-term practice of a variety of balance tasks did not facilitate the learning of a new balance task, underpinning the specificity of balance training effects and the lack of transfer from one balance task to another. The relation between lower limb power and the learning rate suggests that the motor learning of dynamic balance tasks seems to depend on the physical capability to execute the correct movement. Thus, a prior strength and conditioning program with emphasis on lower limb power should be considered when designing a balance training, especially in fall prevention.

\section{Practical implications}

- Practicing several balance tasks before learning a novel dynamic balance task does not seem to improve the learning of that task.

- Participants with high leg power learned the novel balance task faster than those with lower leg power.

- Completing a prior strength and power conditioning program might be beneficial for balance training effects.

\section{Acknowledgements}

The authors want to thank jors Hendrikx and Tyler Breedlove for their help with subject recruitment and data recording and processing.

\section{References}

1. Donath L, Roth R, Zahner L et al. Slackline training (balancing over narrow nylon ribbons) and balance performance: a meta-analytical review. Sports Med 2017; 47(6):1075-1086.

2. Giboin LS, Gruber M, Kramer A. Task-specificity of balance training. Hum Mov Sci 2015; 44:22-31.

3. Kummel J, Kramer A, Giboin LS et al. Specificity of balance training in healthy individuals: a systematic review and meta-analysis. Sports Med 2016; 46(9):1261-1271.

4. Hannan EL, Magaziner J, Wang JJ et al. Mortality and locomotion 6 months after hospitalization for hip fracture: risk factors and risk-adjusted hospital outcomes. JAMA 2001; 285(21):2736-2742.

5. Dargent-Molina P, Favier F, Grandjean H et al. Fall-related factors and risk of hip fracture: the EPIDOS prospective study. Lancet 1996; 348(9021):145-149.

6. Seidler RD. Multiple motor learning experiences enhance motor adaptability. J Cogn Neurosci 2004: 16(1):65-73.

7. Harlow HF. The formation of learning sets. Psychol Rev 1949; 56(1):51-65.

8. Braun DA, Mehring C, Wolpert DM. Structure learning in action. Behav Brain Res 2010; 206(2):157-165.

9. Kattner F, Cochrane A, Cox CR et al. Perceptual learning generalization from sequential perceptual training as a change in learning rate. Curr Biol 2017: 27(6):840-846.

10. Bejjanki VR, Zhang R, Li R et al. Action video game play facilitates the development of better perceptual templates. Proc Natl Acad Sci U S A 2014; 111(47):16961-16966.

11. Brown AL, Kane MJ. Preschool children can learn to transfer: learning to learn and learning from example. Cogn Psychol 1988; 20(4):493-523.

12. Braun DA, Aertsen A, Wolpert DM et al. Motor task variation induces structural learning. Curr Biol 2009; 19(4):352-357.

13. Mulavara AP, Cohen HS, Bloomberg JJ. Critical features of training that facilitate adaptive generalization of over ground locomotion. Gait Posture 2009; 29(2):242-248.

14. Anderson K, Behm DG. The impact of instability resistance training on balance and stability. Sports Med 2005; 35(1):43-53.

15. Paillard T. Relationship between muscle function, muscle typology and postural performance according to different postural conditions in young and older adults. Front Physiol 2017; 8:585.

16. Bates D, Mächler M, Bolker B et al. Fitting linear mixed-effects models using Ime4. J Statistical Softw 2015; 67(1).

17. Kuznetsova A, Brockhoff PB, Christensen RHB. lmerTest package: tests in linear mixed effects models. J Stat Softw 2017; 82(13).

18. Ringhof S, Stein T. Biomechanical assessment of dynamic balance: specificity of different balance tests. Hum Mov Sci 2018; 58:140-147.

19. Horlings CG, van Engelen BG, Allum JH et al. A weak balance: the contribution of muscle weakness to postural instability and falls. Nat Clin Pract Neurol 2008; 4(9):504-515.

20. Orr R. Contribution of muscle weakness to postural instability in the elderly. A systematic review. Eur J Phys Rehabil Med 2010; 46(2):183-220.

21. Muehlbauer T, Besemer C, Wehrle A et al. Relationship between strength, power and balance performance in seniors. Gerontology 2012; 58(6):504-512.

22. Muehlbauer T, Gollhofer A, Granacher U. Relationship between measures of balance and strength in middle-aged adults. J Strength Cond Res 2012; 26(9):2401-2407.

23. Zemkova E, Jelen M, Kovacikova Z et al. Balance performance during perturbed standing is not associated with muscle strength and power in young adults. $J$ Mot Behav 2017; 49(5):514-523.

24. Low DC, Walsh GS, Arkesteijn M. Effectiveness of exercise interventions to improve postural control in older adults: a systematic review and meta-analyses of centre of pressure measurements. Sports Med 2017; 47(1):101-112.

25. Orr R, de Vos NJ, Singh NA et al. Power training improves balance in healthy older adults. J Gerontol A Biol Sci Med Sci 2006; 61(1):78-85.

26. Dowse RA, McGuigan MR, Harrison C. Effects of a resistance training intervention on strength power, and performance in adolescent dancers. J Strength Cond Res 2017. http://dx.doi.org/10.1519/JSC.0000000000002288. PMID: 29112056.

27. Pijnappels M, van der Burg PJ, Reeves ND et al. Identification of elderly fallers by muscle strength measures. Eur J Appl Physiol 2008; 102(5):585-592.

28. Gruber M, Gollhofer A. Impact of sensorimotor training on the rate of force development and neural activation. Eur J Appl Physiol 2004; 92(1-2):98-105. 